\title{
T1 Mapping for Microstructural Assessment of the Cervical Spinal Cord in the Evaluation of Patients with Degenerative Cervical Myelopathy
}

\author{
(D) G. Baucher, (D) H. Rasoanandrianina, (D) S. Levy, (D). Pini, (D). Troude, (DP.-H. Roche, and (D). Callot
}

\begin{abstract}
BACKGROUND AND PURPOSE: Although current radiologic evaluation of degenerative cervical myelopathy by conventional MR imaging accurately demonstrates spondylosis or degenerative disc disease causing spinal cord dysfunction, conventional MR imaging still fails to provide satisfactory anatomic and clinical correlations. In this context, we assessed the potential value of quantitative cervical spinal cord T1 mapping regarding the evaluation of patients with degenerative cervical myelopathy.
\end{abstract}

MATERIALS AND METHODS: Twenty patients diagnosed with mild and moderate-to-severe degenerative cervical myelopathy and 10 healthy subjects were enrolled in a multiparametric MR imaging protocol. Cervical spinal cord T1 mapping was performed with the MP2RAGE sequence procedure. Retrieved data were processed and analyzed regarding the global spinal cord and white and anterior gray matter on the basis of the clinical severity and the spinal canal stenosis grading.

RESULTS: Noncompressed levels in healthy controls demonstrated significantly lower $\Pi$ values than noncompressed, mild, moderate, and severe stenotic levels in patients. Concerning the entire spinal cord $\Pi 1$ mapping, patients with moderate-to-severe degenerative cervical myelopathy had higher $\Pi$ values compared with healthy controls. Regarding the specific levels, patients with moderate-to-severe degenerative cervical myelopathy demonstrated a $\mathrm{Tl}$ value increase at $\mathrm{Cl}, \mathrm{C} 7$, and the level of maximal compression compared with healthy controls. Patients with mild degenerative cervical myelopathy had lower $\mathrm{T}$ values than those with moderate-to-severe degenerative cervical myelopathy at the level of maximal compression. Analyses of white and anterior gray matter confirmed similar results. Strong negative correlations between individual modified Japanese Orthopaedic Association scores and $\Pi$ values were also observed.

CONCLUSIONS: In this preliminary study, 3D-MP2RAGE $\Pi$ mapping demonstrated increased $T$ values in the pathology tissue samples, with diffuse medullary alterations in all patients with degenerative cervical myelopathy, especially relevant at $\mathrm{Cl}$ (nonstenotic level) and at the maximal compression level. Encouraging correlations observed with the modified Japanese Orthopaedic Association score make this novel approach a potential quantitative biomarker related to clinical severity in degenerative cervical myelopathy. Nevertheless, patients with mild degenerative cervical myelopathy demonstrated nonsignificant results compared with healthy controls and should now be studied in multicenter studies with larger patient populations.

ABBREVIATIONS: $\mathrm{DCM}=$ degenerative cervical myelopathy; $\mathrm{Cmax}=$ maximal compression; $\mathrm{HC}=$ healthy controls; mJOA = modified Japanese Orthopaedic Association; OSS = overall stenosis score; $\mathrm{SC}=$ spinal cord

D egenerative cervical myelopathy (DCM) currently covers the various cervical spine pathologic conditions potentially causing spinal cord (SC) impairment, including cervical spondylosis and degenerative disc disease. ${ }^{1}$ The wide range of severity of clinical

Received March 11, 2020; accepted after revision February 7, 2021.

From the Neurochirurgie Adulte (G.B., L.T., P.-H.R.), Assistance Publique-Hôpitaux de Marseille, Hôpital Universitaire Nord, Marseille, France; Center for Magnetic Resonance in Biology and Medicine (G.B., H.R., L.P., S.L., V.C.), Assistance PubliqueHôpitaux de Marseille, Hôpital Universitaire Timone, Marseille, France; Center for Metabolic Exploration in Biology and Medicine (H.R., L.P., S.L., V.C.), Aix-Marseille Université, Centre National de la Recherche Scientifique, Marseille, France; and iLab-Spine International Associated Laboratory (G.B., H.R., S.L., P.-H.R., V.C.), Marseille-Montreal, France-Canada.

This work was supported by the Institut Carnot Star and Centre National de la Recherche Scientifique. presentations makes the disability caused by DCM widely variable but usually substantially associated with a reduction in the quality of life. ${ }^{2,3}$ DCM is generally considered the first cause of SC dysfunction among adults, ${ }^{4}$ and the epidemiology of DCM remains complex to estimate because of frequent diagnostic delays. ${ }^{1}$ Cervical spondylosis and degenerative disc disease are currently found in approximately $70 \%$ of individuals older than 65 years of age and $70 \%$ of

Please address correspondence to Virginie Callot, PhD, CRMBM-CEMEREM, UMR7339, CNRS, Aix-Marseille Université, 27 Boulevard Jean Moulin, 13385 Marseille Cedex 05, France; e-mail: virginie.callot@univ-amu.fr

\footnotetext{
- Indicates open access to non-subscribers at www.ajnr.org

Indicates article with online supplemental data.

http://dx.doi.org/10.3174/ajnr.A7157
} 
asymptomatic individuals. ${ }^{5,6}$ In addition to a disposition to a further evolution toward progressive symptomatic myelopathy, these conditions also expose patients to the risk of acute neurologic deterioration in cases of traumatic hyperextension or hyperflexion, notably illustrated by the central cord syndrome. ${ }^{7-9}$

Once the diagnosis of DCM is suspected, the role of MR imaging is obviously useful for demonstrating cervical spinal degenerative changes and signs of SC impairment. ${ }^{10}$ First, MR imaging provides direct information about spinal canal size and the structural causes responsible for its narrowing. ${ }^{11}$ Second, conventional T2WI and T1WI highlight intramedullary signal intensity changes, which are associated with postoperative functional outcome. ${ }^{10}$

Nevertheless, the major discrepancy between clinical severity and the radiologic degree of stenosis is a important issue for the neurosurgeon. Several advanced MR imaging techniques have, thus, been proposed as potential biomarkers of DCM, attempting to more accurately assess the microstructural and functional organization of the SC. DTI, measuring directional diffusivity of water within each voxel, ${ }^{12,13}$ appears to be the most studied and promising technique. However, it currently remains complex to apply in daily practice due to difficulties in establishing standardized protocols, such as an appropriate anatomic level of acquisition and surgery-predictive cutoff values. ${ }^{14}$

Although longitudinal relaxation time $\mathrm{T} 1$ is an essential parameter in MR imaging, T1 mapping has not been used in clinical routine practice due to its long scan time. Due to faster techniques of acquisition, ${ }^{15-18}$ it now appears as another option to help analyze pathologic SC and, more particularly highly myelinated tissue associated with low T1 values. ${ }^{19-21}$ Water content, axonal size, and iron concentration also influence T1, explaining the sensitivity of this approach to pathologic microstructural changes in neural tissue, despite a lack of specificity. ${ }^{22-24}$ Used in the brain to study alterations in white and gray matter in both lesions and tissue with a normal appearance, T1 values demonstrated an interesting clinical correlation with cognitive dysfunction. ${ }^{25,26}$ Although it has been rarely used in the SC until recently, studies have now outlined fast and reproducible methods to measure T1 in cervical SC in healthy subjects using 3D-MP2RAGE, ${ }^{27} 2 \mathrm{D}$ multisection inversion recovery zonally oblique-magnified multislice $\mathrm{EPI}{ }^{18}$ or $2 \mathrm{D}$ single-section inversion recovery radial gradient-echo ${ }^{28}$ sequences. This latter procedure, recently applied in patients presenting with mild and moderate cervical spinal canal stenosis, ${ }^{28}$ demonstrated decreased $\mathrm{T} 1$ values in the SC at the compression site, with a more pronounced decrease in the higher-grade stenosis. To the best of our knowledge, this is, to date, the only quantitative T1 investigation in a pathologic context.

The aim of the current study was, therefore, to go further and pursue the investigation of microstructural alterations in patients with diagnosed DCM, by performing routine T1 mapping of the entire cervical SC (ie, not restricted to the level of maximal compression) using the 3D-MP2RAGE sequence (for the first time in the pathologic SC) and evaluating both white and gray matter compartments (instead of the SC alone). In this study, we compared T1 values collected in the healthy cervical SC with compressed levels and levels of normal appearance in patients with DCM, and using the correlation with clinical features, we explored the potential diagnostic contribution of T1 mapping.

\section{MATERIALS AND METHODS}

\section{Subject Recruitment and Clinical Assessment}

In this prospective single-center study, 20 volunteer patients presenting with clinical and radiologic signs of DCM were recruited in an adult neurosurgery department (Hôpital Nord, AP-HM). Ten healthy subjects with no clinical signs of myelopathy were enrolled in parallel as a control group. Exclusion criteria were contraindications for MR imaging and intolerance of the supine position. The study was approved by the local ethics committee (South Mediterranean Committee for the Protection of Individuals), and written informed consent was routinely obtained before MR imaging for all patients and healthy controls (HC). Clinical presentation was assessed using the modified Japanese Orthopaedic Association (mJOA) scale, with scores ranging from 0 to $17{ }^{29}$ Patients were separated into mild DCM (mJOA score from 15 to 17 ) and moderate-to-severe DCM (mJOA score of $\leq 14$ ) groups. ${ }^{30}$ The clinical onset of the symptoms was classified as acute or chronic.

\section{MR Imaging Protocol}

MR imaging examinations were performed on a 3T system (Magnetom Verio; Siemens) using standard 12-channel head, 4channel neck, and 24-channel spine matrix coils for signal reception. Subjects were placed in a supine position with the neck fixed in a neutral position using a cervical brace to prevent any movement during the procedure. The protocol included a 3D T2 sagittal sampling perfection with application-optimized contrasts by using different flip angle evolution (SPACE sequence; Siemens) and MP2RAGE acquisitions. The MP2RAGE ${ }^{31}$ sequence was optimized for the SC as in Rasoanandrianina et $\mathrm{al}^{27}$ ( $\mathrm{TE}=2.48 \mathrm{~ms}$, $\mathrm{TR}=4$ seconds, generalized autocalibrating partially parallel acquisition $=2$, isotropic resolution $=0.9 \mathrm{~mm}$ ) and covered both brain and C1-C7 vertebral levels (Fig 1). This sequence provided both anatomic imaging and quantitative T1 mapping. In fact, normally the MP2RAGE sequence acquires two 3D gradient-echo volumes at 2 different $\mathrm{TIs}\left(\mathrm{T} 1_{\text {inv1 }}\right.$ and $\mathrm{T} 1_{\text {inv2 }}$, here 65 and $\left.2000 \mathrm{~ms}\right)$ with 2 different flip angles $\left(\alpha_{1}\right.$ and $\alpha_{2}$, here $4^{\circ}$ and $\left.5^{\circ}\right)$ that are combined to obtain a composite "uniform" T1WI contrasted volume, called $\mathrm{T} 1_{\mathrm{UNI}}{ }^{27}$ from which a $\mathrm{T} 1$ map is subsequently derived. Because $T 1_{\mathrm{UNI}}$ signal is partially altered by the transmitted $\mathrm{B}_{1}{ }^{+}$ and received $\mathrm{B}_{1}$ - field inhomogeneities (bias fields), leading to inaccurate $\mathrm{T} 1$ estimation, an additional magnetized-prepared turbo-FLASH $\mathrm{B}_{1}{ }^{+}$mapping sequence covering the whole cervical $\mathrm{SC}$ was acquired to correct bias fields during postprocessing. The total acquisition time was approximately 15 minutes.

\section{Data Postprocessing}

The data postprocessing aimed at extracting corrected $\mathrm{T} 1$ values from $\mathrm{Tl}_{\mathrm{UNI}}$ acquisitions, at the level of the cervical SC, using the $\mathrm{T}_{\text {inv2 }}$ sequence as an anatomic reference. The postprocessing stages were performed using in-house Matlab codes (R2018a version; MathWorks). $\mathrm{T} 1_{\mathrm{UNI}}$ and $\mathrm{B}_{1}{ }^{+}$maps were first combined to offset bias fields, generating the adjusted $\mathrm{T} 1_{\mathrm{Q}}{ }^{27}$ Denoising of $\mathrm{T} 1_{\mathrm{Q}}$ was achieved by Block-matching $4 \mathrm{D}$ filtering (BM4D). ${ }^{32}$ The next stage consisted of cervical SC segmentation using the semi-automated processing pipeline PropSeg, ${ }^{33}$ included in the Spinal Cord Toolbox. $^{34}$ Due to frequent difficulties of PropSeg in 

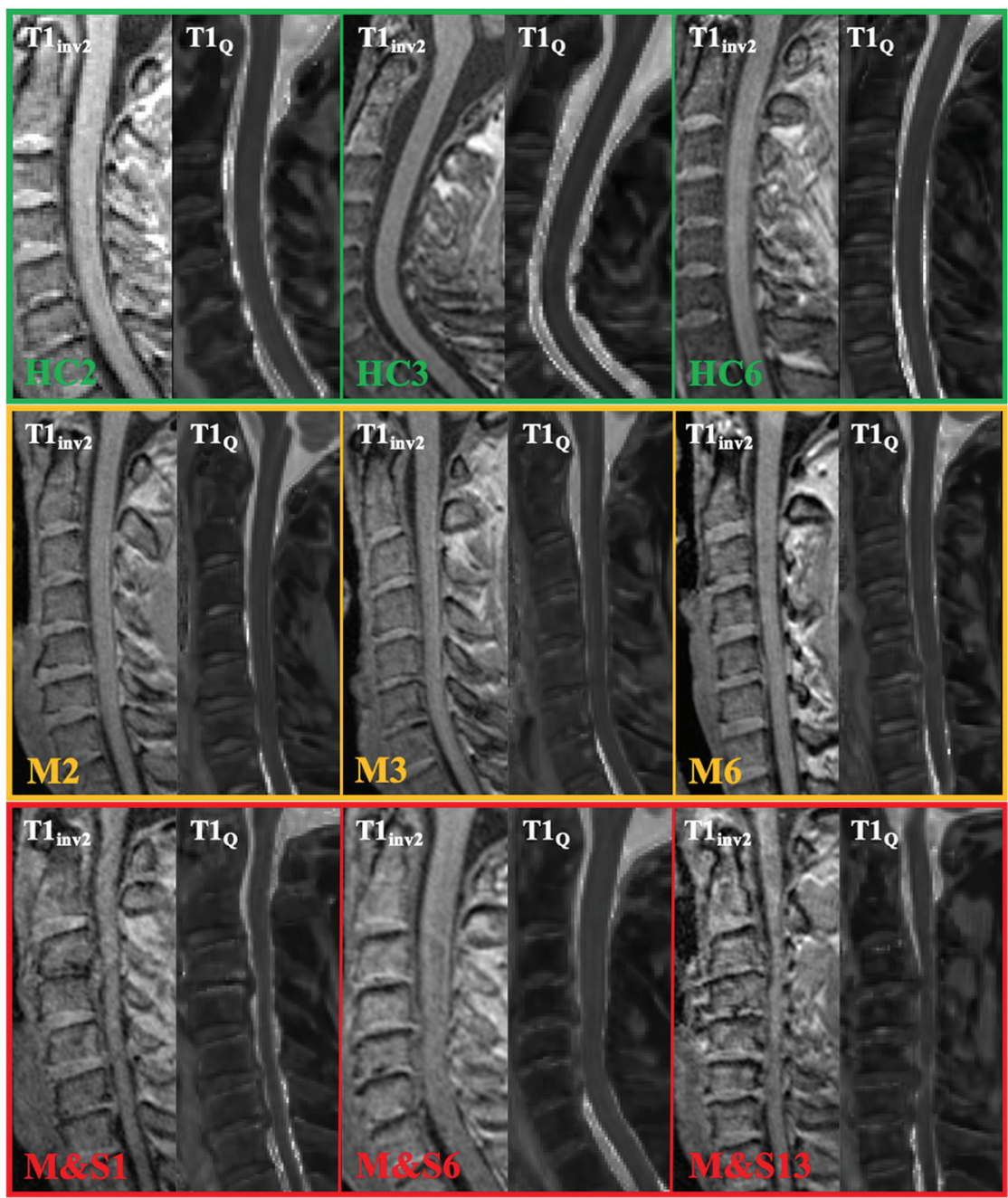

FIG 1. Illustrative cases of cervical sagittal MP2RAGE views $\left(\Pi_{\text {inv2 }}\right.$ and $\left.T_{\mathrm{Q}}\right)$ acquired in healthy controls (upper row), patients with mild DCM (middle row), and moderate-to-severe DCM (lower row). $M$ indicates mild; $M \& S$, moderate and severe. correctly projecting the SC beyond compressed levels, the obtained cord segmentation from the $\mathrm{T} 1_{\text {inv2 }}$ volume was systematically reviewed and manually corrected to match both $\mathrm{T} 1_{\mathrm{Q}}$ and $\mathrm{T} 1_{\text {inv2 }}$ (Fig 2). The $\mathrm{T} 1_{\mathrm{Q}}$ volume and its corresponding cord segmentation were then registered into the anatomic and probabilistic PAM50 space. $^{35,36}$ Vertebral labeling was derived from this process and routinely checked visually. SC GM, including anterior, intermediate, and dorsal GM, and WM delineations were also obtained using the PAM50 atlases. The binarization of the GM/WM segmentation was completed using 0.5 as a threshold for each voxel. Warping back of the cord segmentation and WM/GM masks into the subject space allowed new visual checking and correction in case of an anatomic defect. Using these masks and following a $2 \mathrm{D}$ erosion around the cord, we quantified T1 values within the entire SC, WM, and anterior and intermediate GM for each vertebral level from C1 to C7. Anterior and intermediate GM ROIs were merged into a single ROI. Posterior GM was intentionally excluded from the analysis to avoid a substantial risk of partial volume effects and erroneous results due to the small dimensions of the posterior horns at the

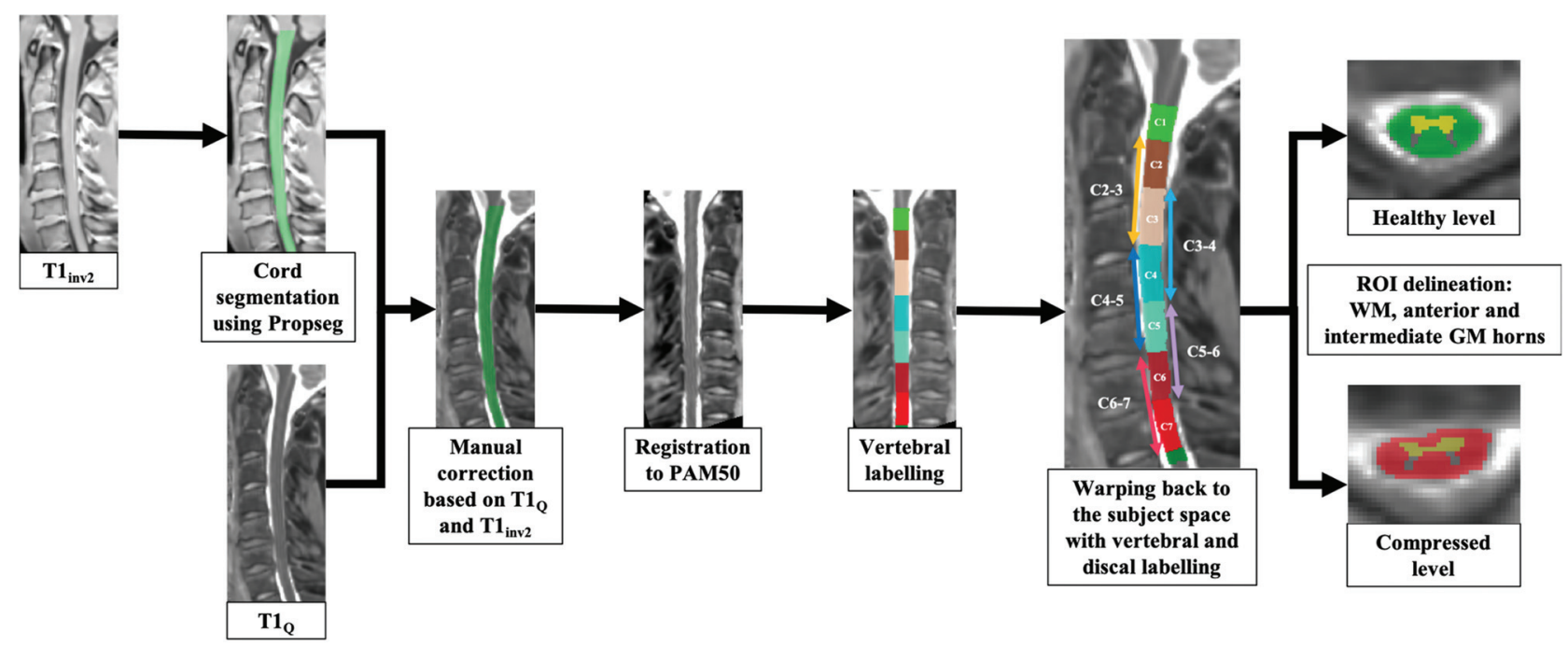

FIG 2. Postprocessing of the T1 data from cord segmentation to WM/GM ROI analysis, per vertebral and disc levels. A Tl $\mathrm{Q}$ threshold of 2 seconds was used to check the cord segmentation. Segmentation was performed using the PAM50 atlas. ${ }^{34,35}$ Quantifications were performed in the subject space. 
cervical level regarding the spatial resolution of the images at $3 \mathrm{~T}$.

\section{Anatomic Assessment of the Spinal Stenosis}

Compression of the SC was assessed using axial and sagittal views of the anatomic T2-SPACE series, as usual in daily practice. We selected a qualitative scale of spinal canal stenosis, presenting satisfactory intraobserver and interobserver reliabilities (Online Supplemental Data). ${ }^{37,12}$ This grading, ranging from 0 to 3 , classified each disc level as normal, mildy, moderately, or severely compressed, respectively. The level of maximal compression was determined for each patient and termed "Cmax." These observations were routinely rated by an experienced neurosurgeon, blindly, from the clinical severity and before T1-mapping postprocessing. An overall stenosis score (OSS) was additionally proposed for each subject by adding the single stenosis scores (from 0 to 3) for each disc level from $\mathrm{C} 2-\mathrm{C} 3$ to $\mathrm{C} 6-\mathrm{C} 7$. The obtained value, ranging from 0 to 15 , was then used to define 3 grades of OSS severity based on the statistical distribution of the results: mild OSS (0-6) (first quartile, 5.8); moderate OSS (7-9); and severe OSS (10-15) (third quartile, 10). The presence of T2 signal hyperintensity at the level of maximal compression was evaluated as well.

\section{Statistical Analysis}

All statistical analyses were performed using JMP 9 (SAS Institute), considering $P$ values $<.05$ as statistically significant. T1 values at the disc levels ( $\mathrm{C} 2-\mathrm{C} 3$ to $\mathrm{C} 6-\mathrm{C} 7)$ were calculated by averaging the 2 adjacent vertebral level T1 values from $\mathrm{C} 2$ to $\mathrm{C} 7$ for each subject. Due to the large number of compressed levels in both the mild and moderate-to-severe groups of patients, we decided to compare $\mathrm{T} 1$ values for C1, C7, and Cmax. Because C4-C5 and C5-C6 were the most frequent levels of maximal compression (respectively, $40 \%$ and $40 \%$ of all patients), we took the T1 value of the vertebral C5 level into account for the healthy subjects' group, to compare with the Cmax results of the groups of patients. T1 comparisons between the different groups (clinical severity, clinical onset, spinal canal stenosis) were performed using a linear analysis of covariance (age as a covariate), followed by a nonparametric Steel-Dwass test corrected for multiple comparisons. Age effect was reported when appropriate. The Spearman coefficients were calculated to study the correlation between clinical severity (preoperative mJOA score) and $\mathrm{T} 1$ values.

\section{RESULTS}

\section{Epidemiology}

The healthy control group included 10 subjects (mean, 45.1 [SD, 17.7] years of age; range, 22-66 years); the mild DCM group, 7 patients (mean, 41.3 [SD, 15.2] years of age; range, 25-71 years; mean $\mathrm{mJOA}, 16.3$ [SD, 0.5]; range, 16-17); and the moderate-tosevere group, 13 patients (mean, 54.0 [SD, 14.2] years; range, 30-72 years; mean mJOA, 12.5 [SD, 1.7]; range, 9-14). There was no statistical difference concerning age among these 3 clinical groups. Four elderly subjects and 1 young healthy subject had moderate radiologic compression (grades 1-2) related to degenerative changes but remained in the control group due to their absence of symptoms and clinical signs of myelopathy. Details are presented in the Online Supplemental Data. The maximal compression was primarily found at C5-C6 in the mild DCM group (71\%) and at C4C5 in the moderate-to-severe DCM group (54\%). The mean number of compressed disc levels was 3.7 (SD, 1.1): 3.3 (SD, 1.6) in the mild DCM group and 3.8 (SD, 0.8) in the moderate-to-severe DCM group. The mean spinal canal stenosis score (from 0 to 3 for each disc level) for the compressed levels was 2.0 (SD, 0.9) for the mild DCM group, 2.1 (SD, 0.9) for the moderate-to-severe DCM group, and 2.9 (SD, 0.3) at Cmax (equal in both patient groups). Six patients $(30 \%, 49.3[\mathrm{SD}, 13.4]$ years of age on average) had an acute onset of their neurologic signs, whereas 14 patients (70\%, 49.6 [SD, 16.7] years of age) had a progressive appearance of their symptoms. Cervical spondylosis was the predominant cause of DCM, observed in 15 patients (75\%) compared with 5 patients with degenerative disc disease (25\%). Patients affected with degenerative disc disease were significantly younger (35.8 [SD, 7.5] years of age on average) than those with spondylosis (54.1 [SD, 14.8] years of age, $P=.02$ ). Nevertheless, the mJOA score did not differ in the 2 etiologic entities (mean mJOA: 14.6 [SD, 2.8] for degenerative disc versus 13.6 [SD, 2.2] for spondylosis).

\section{T1 Mapping and Clinical Onset}

The mJOA score between the patients presenting with an acute onset of their symptoms $(n=6$, mean $\mathrm{mJOA}=13.7[\mathrm{SD}, 2.5])$ and those having a progressive neurologic course $(n=14$, mean $\mathrm{mJOA}=13.9$ [SD, 2.3]) did not show any significant difference. Despite a slightly superior vertebral distribution of T1 values for acute onset, statistical analyses failed to show any significant difference between the 2 clinical entities at C1 (mean T1 for acute onset, 977.1 [SD, 36.4] ms; and chronic onset, 964.0 [SD, 27.3] ms), Cmax (1001.6 [SD, 88.4] ms and 981.0 [SD, $63.3] \mathrm{ms})$, and C7 $(981.8[\mathrm{SD}, 47.6] \mathrm{ms}$ and 958.0 [SD, 49.6] ms) levels.

\section{T1 Mapping and Cervical Spinal Canal Stenosis}

When we compared noncompressed disc level T1 values in healthy controls (grade 0) with all disc level T1 values in patients (grade 0-3), an overall increased T1 value was observed for all patient grades (Fig 3), with a significant difference between healthy subject grade 0 and patient grade $0(P<.001)$, grade $1(P=.003)$, grade $2(P=.03)$, and grade $3(P<.001$, with an age effect $(P=.04)$. Overall (all subjects combined) increased T1 values in the cervical SC were observed in cases of spinal canal stenosis, with statistically significant differences between grades 3 and $0(P<.001$, age effect $[P=.047])$ and grades 3 and 1 $(P<.05)$.

\section{T1 Mapping and T2 Hyperintensity}

Among the 20 patients, 10 had medullary T2 hyperintensity (Online Supplemental Data), corresponding to a mean T1 value of 1037 [SD, 64] $\mathrm{ms}$ at the level of maximal compression (median $=1039 \mathrm{~ms}$; minimum-maximum $=949-1157 \mathrm{~ms})$. In comparison, the remaining 10 patients having a normal medullary T2 intensity had a statistically significant lower mean T1 value of $937(\mathrm{SD}, 25) \mathrm{ms}$ (median $=935 \mathrm{~ms}$; minimum-maximum $=905-990 \mathrm{~ms})(P<.001)$. 


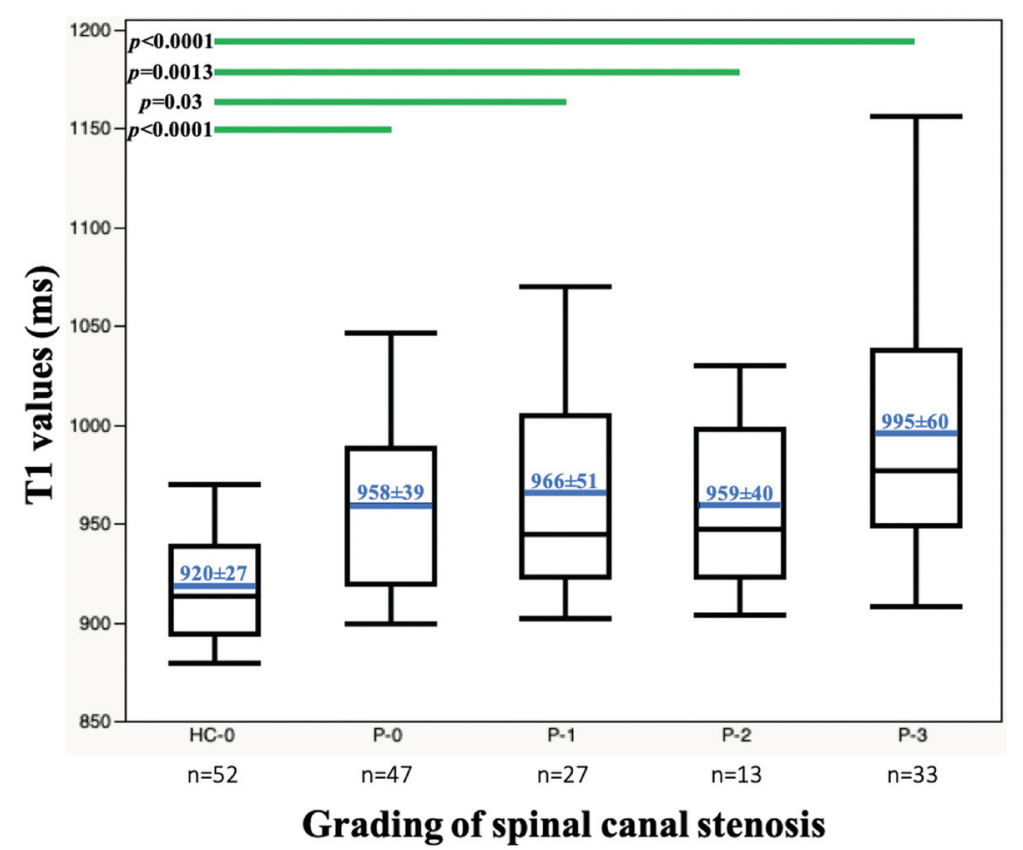

FIG 3. Boxplots of cervical spinal cord $T\rceil$ values (in milliseconds) in noncompressed disc levels for the 10 healthy controls (HC-0 grade 0; mean T1, 920 [SD, 27] ms) compared with noncompressed (P-0 grade 0; mean T1, 958 [SD, 39] ms), mildly (P-1 grade I; mean T1, 966 [SD, 51] ms), moderately (P2 grade II; mean T1, 959 [SD, 40] ms), and severely (P-3 grade III; mean Tl, 995 [SD, 60] ms) compressed disc levels for the 20 patients. $\mathrm{Cl}$ and $\mathrm{C} 2-\mathrm{C} 3$ to $\mathrm{C} 6-\mathrm{C} 7$ disc levels were considered for each subject ( 6 levels in total). Grade 0 in patients and healthy controls were considered separately because they present statistical different values. The horizontal blue lines represent the mean value in each group. The horizontal lines within and at the ends of the boxes represent the median value and the first and third quartiles, respectively. The whiskers illustrate the minimum and maximum values. The horizontal green lines demonstrate statistically significant differences between the stenosis grades.

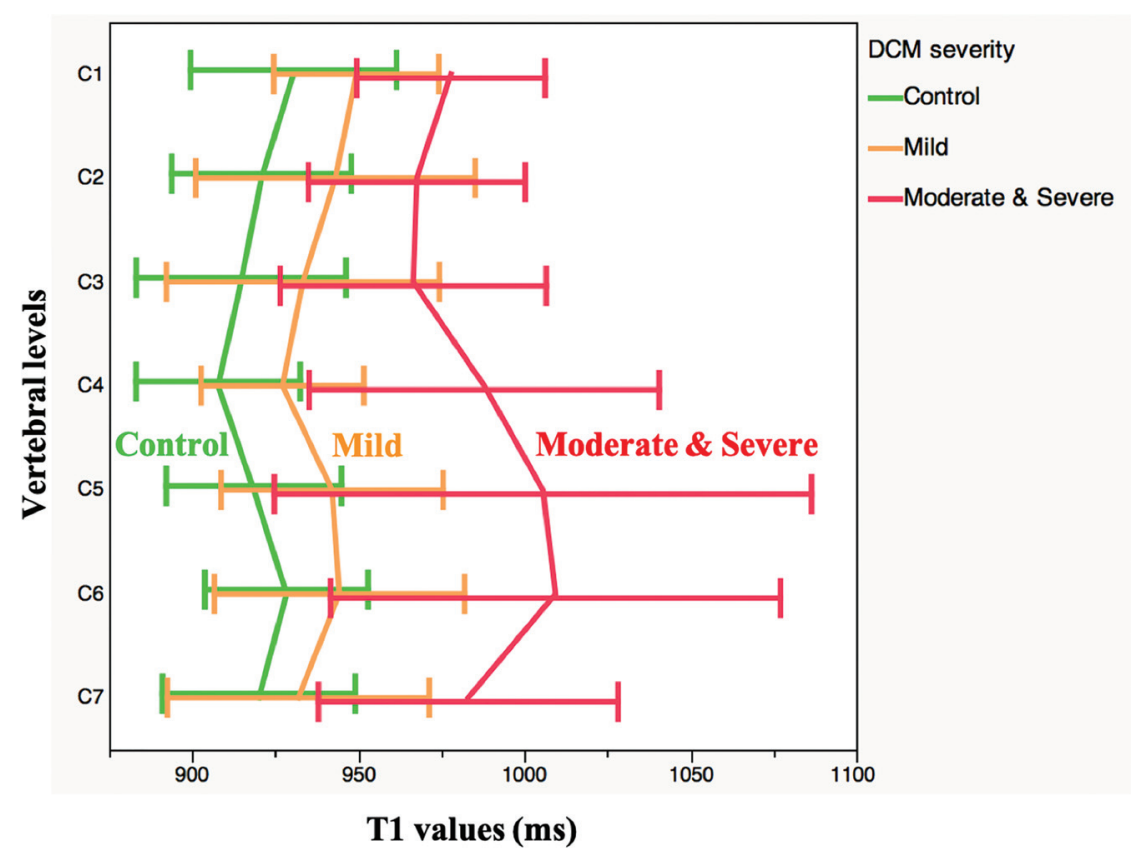

FIG 4. Distribution of the cervical spinal cord $\Pi 7$ values (milliseconds) according to the vertebral levels in the healthy subjects (green, no statistical difference between the different cervical levels) and patients with mild (orange) and moderate-to-severe (red) DCM. The horizontal bars represent the SD for each level.

\section{Global TI Mapping and Overall Stenosis Score}

Statistical analysis of the global SC T1 values in the 20 patients (single value for each subject, calculated by the mean T1 value for the whole cervical SC), regarding the OSS, demonstrated lower values in the mild OSS group (OSS = 1-6, seven patients; mean T1, 936.9 [SD, 35.7] ms) compared with the highest outcomes of the moderate OSS group (OSS = 7-9, nine patients; mean, 988.7 [SD, 40.9] ms) and with the severe OSS group (OSS $=10-15$; four patients; mean, 968.6 [SD, 14.2] ms). Statistical analyses were not conclusive regarding differentiating the severe OSS group from the mild and moderate groups, though comparison between the moderate and the mild groups was significant $(P=.02)$.

\section{T1 Mapping and Clinical Severity}

Cervical SC T1 mapping in the 3 clinical groups revealed increased $\mathrm{T} 1$ values in the moderate-to-severe group compared with the mild DCM group and healthy subjects (Fig 4). In comparison, the mild DCM group demonstrated slightly higher cord T1 distribution compared with the healthy control group. Global inflection of the $3 \mathrm{~T} 1$ curves could be observed around the $\mathrm{C} 3$ and $\mathrm{C} 4$ levels (but no statistical difference was observed among the different vertebral levels). Considering specifically the single global SC T1 value for each patient in the 3 clinical groups, patients with moderate-to-severe DCM had the highest mean SC T1 value (982.1 [SD, 39.4] ms), followed by the patients with mild DCM (937.7 [SD, 32.9] ms), and healthy subjects (920.1 [SD, 24.1] ms). A significant difference was found between the patients with moderate-to-severe DCM and healthy subjects $(P=.002)$. T1 values in the mild DCM and moderate-tosevere DCM groups were close to statistical significance $(P=.06)$, whereas no difference was observed between the patients with mild DCM and healthy subjects $(P=.50)$. Finally, a significant correlation between the single SC T1 values and the preoperative mJOA scores was observed ( $\rho=$ -0.68 and $P=.003$ ). 


\section{Level-Specific T1 Mapping and Clinical Severity}

When we compared the SC T1 values at the C1, Cmax, and C7 levels among the different clinical groups, a global increase was observed from the control group to the mild and then moderate-to- severe DCM groups (Fig 5). Statistical analysis revealed significant differences between healthy subjects and the moderate-to-severe DCM group at each level $(P=.005, .003$, and .007, at C1, Cmax, and $\mathrm{C7}$, respectively). The relationships between the mild and

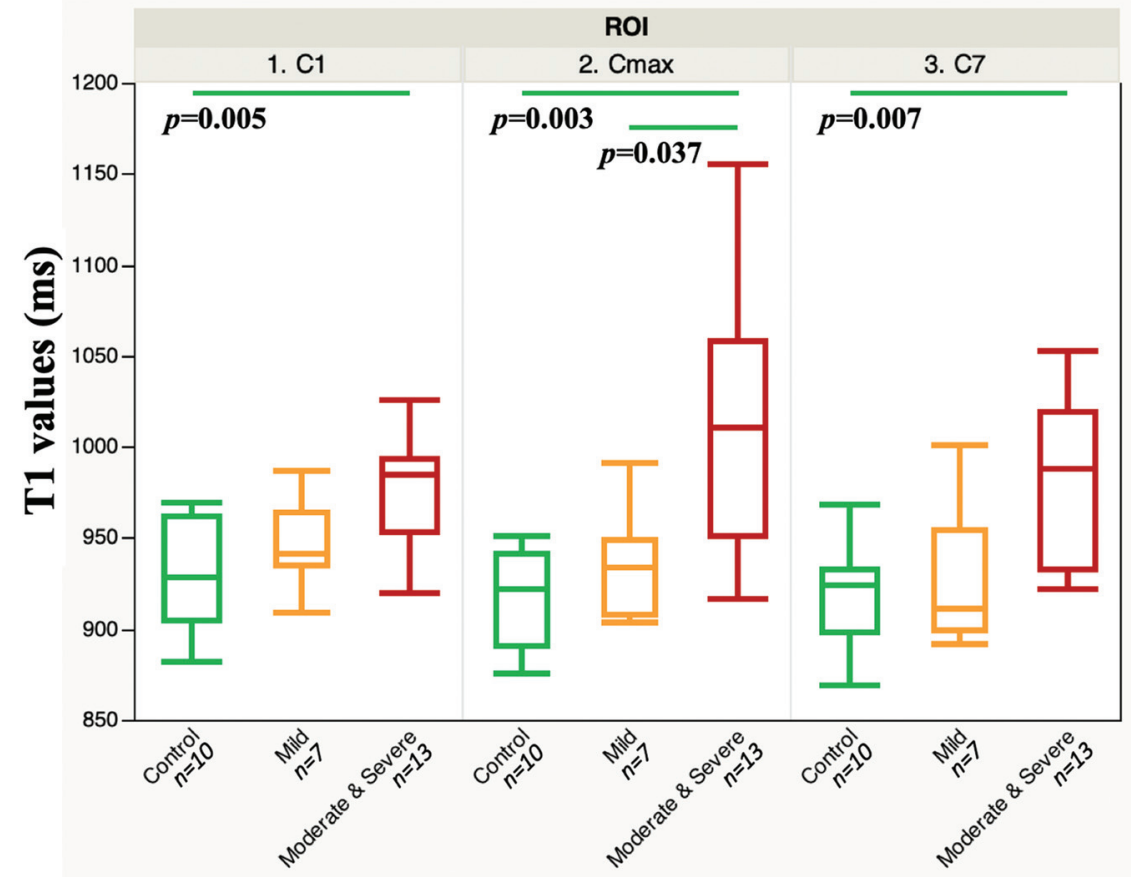

Clinical severity

FIG 5. Boxplots of the $\mathrm{Tl}$ values at $\mathrm{Cl}, \mathrm{Cmax}$, and $\mathrm{C} 7$ in the control (green), mild (orange), and moderate-to-severe (red) DCM groups. Horizontal green lines represent statistically significant differences among the clinical groups $(P<.05)$.

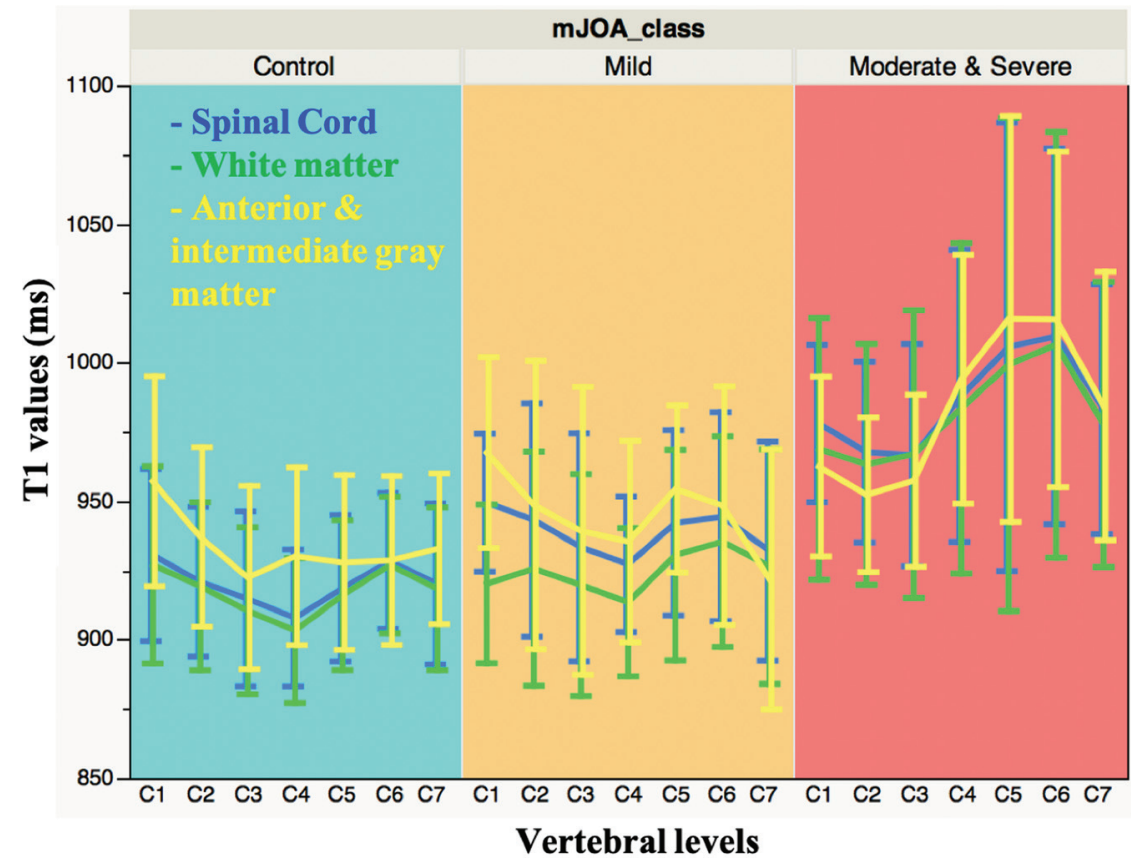

FIG 6. Distribution of the $T 7$ values (milliseconds) for each clinical group according to the vertebral levels, in the entire spinal cord (blue), white matter (green), and anterior gray matter (yellow). Vertical bars represent the SD for each level. the moderate-to-severe DCM groups showed a significant difference at Cmax $(P=.04)$. Comparisons between the healthy subjects and the mild DCM group failed to show any statistical relationship. When we considered individual $z$ scores (each patient's T1 relative to the mean of healthy controls) at Cmax, 10/13 patients with moderateto-severe DCM had values of $>2$ (12/ 13 had $z$ scores of $>1$ ). For the patients with mild DCM, 4/7 had $z$ scores of $>1$, but only one was above 2 . Correlations between the preoperative mJOA score and $\mathrm{T} 1$ values at $\mathrm{C} 1$, Cmax, and C7 were also assessed using the Spearman coefficient and were consistently significant $(\rho=-0.63$ and $P=.01 ; \rho=-0.71$ and $P=.002 ; \rho=$ -0.62 and $P=.01$ ).

\section{White Matter and Anterior Gray Matter T1 Mapping and Clinical \\ Severity}

Regarding the T1 mapping in more specific anatomic subdivisions, increased values were observed in the anterior and intermediate GM compared with WM and the entire SC in the healthy subjects (Fig 6). Anterior and intermediate GM T1 values remained the highest in the mild DCM group, with values close to those observed in the SC. Finally, anatomic distribution values in the moderate-to-severe DCM group appeared more erratic, without any clear hierarchic organization, but with very high values for both WM and GM, especially in the lower levels. Statistical comparisons of $\mathrm{T} 1$ values at the $\mathrm{C} 1$, Cmax, and C7 levels between the clinical groups globally demonstrated significant differences between healthy subjects and the moderate-tosevere group in $\mathrm{WM}$ at $\mathrm{Cmax}$ $(P=.01)$ and $\mathrm{C} 7(P<.05)$, as well as at Cmax in the anterior GM $(P=.002)$. Results between patients with mild and moderate-to-severe DCM were conclusive at $\mathrm{C} 1$ $(P<.05)$ and $\operatorname{Cmax}(P=.04)$ for $\mathrm{WM}$ and at $\mathrm{Cmax}(P=.01)$ for anterior GM. None of the analyses 
Summary of the global observations and statistical analyses according to radiologic/clinical settings and regionalized T1-mapping

\begin{tabular}{|c|c|c|c|}
\hline Radiologic and Clinical Settings & $\begin{array}{l}\text { Regionalized T1 } \\
\text { Mapping }\end{array}$ & Global Observations & Statistical Significance \\
\hline Clinical onset & $\mathrm{SC}$ at $\mathrm{Cl}, \mathrm{Cmax}, \mathrm{C} 7$ & $\mathrm{~T} 7$ values acute $>$ chronic & No \\
\hline Cervical spinal canal stenosis & SC at disc levels & $\begin{array}{l}\mathrm{T} 1 \text { values increase according to the } \\
\text { severity of the stenosis }\left(T 1_{\text {grade0 }}<\right. \\
\left.T 1_{\text {gradel }}<T 1_{\text {grade3 }}\right)\end{array}$ & $\begin{array}{l}\text { Grades } 3 / 0 ; 3 / 1 \text { (considering all } \\
\text { subjects) } \\
\text { Grades } 3 / 0_{\mathrm{HC}}, 2 / 0_{-} \mathrm{HC}, 1 / 0_{-} \mathrm{HC} \\
0 / 0_{-} \mathrm{HC} \text { (differentiating } \\
\text { patients and } \mathrm{HC} \text { grade } 0 \text { ) }\end{array}$ \\
\hline Oss & Entire cervical SC & $\begin{array}{l}\text { Lower } \mathrm{Tl} \text { values in the mild-stenosis } \\
\text { group }\end{array}$ & Mild-vs-moderate OSS \\
\hline \multirow[t]{5}{*}{ Clinical severity } & Entire cervical SC & $\begin{array}{l}\mathrm{T} 1 \text { values increase according to the } \\
\text { clinical severity } \\
\left(\mathrm{T}_{\text {control }}<\mathrm{T}_{\text {mild }}<\mathrm{T} 1_{\text {moderate-to-severe})}\right. \\
\text { Inverse relationship between global } \mathrm{T1} \\
\text { values and preoperative mJOA } \\
\text { scores }\end{array}$ & $\begin{array}{l}\text { HC and patients with moderate- } \\
\text { to-severe DCM } \\
\text { Yes }\end{array}$ \\
\hline & $\mathrm{SC}$ at $\mathrm{Cl}, \mathrm{Cmax}, \mathrm{C} 7$ & $\begin{array}{l}\mathrm{T1} \text { values increase at } \mathrm{Cl}, \mathrm{Cmax}, \mathrm{C7} \\
\text { according to the clinical severity }\end{array}$ & $\begin{array}{l}\text { HC and patients with moderate- } \\
\text { to-severe } \mathrm{DCM}(\mathrm{Cl}, \mathrm{Cmax}, \\
\text { and } \mathrm{C} 7) \\
\text { Patients with mild and } \\
\text { moderate-to-severe DCM } \\
\text { (Cmax) } \\
\text { No statistical difference } \\
\text { between controls and } \\
\text { patients with mild DCM }\end{array}$ \\
\hline & $\begin{array}{l}\text { WM, anterior and } \\
\text { intermediate GM at } \\
\mathrm{Cl}, \mathrm{Cmax}, \mathrm{C7}\end{array}$ & $\begin{array}{l}\text { Global Tl-value increase at } \mathrm{Cl}, \mathrm{Cmax} \text {, } \\
\mathrm{C} 7 \text { in the } \mathrm{WM} \text { and } \mathrm{GM} \text { according to } \\
\text { the clinical severity }\end{array}$ & $\begin{array}{l}\mathrm{HC} \text { and patients with moderate- } \\
\text { to-severe } \mathrm{DCM}(\mathrm{Cmax} \text { and } \mathrm{C7} \\
\text { in } \mathrm{WM} \text { and } \mathrm{Cmax} \text { in } \mathrm{GM})\end{array}$ \\
\hline & & & $\begin{array}{l}\text { Patients with mild and } \\
\text { moderate-to-severe } \mathrm{DCM}(\mathrm{Cl} \\
\text { and } \mathrm{Cmax} \text { in WM, Cmax in } \\
\mathrm{GM})\end{array}$ \\
\hline & & & $\begin{array}{l}\text { No statistical difference } \\
\text { between controls and } \\
\text { patients with mild DCM }\end{array}$ \\
\hline
\end{tabular}

between the patients with mild DCM and HC were statistically significant.

\section{DISCUSSION}

T1 values are known to be directly affected by the degree of myelination, axonal diameter, and water content, ${ }^{24}$ with the shorter $\mathrm{T} 1 \mathrm{~s}$ being associated with healthy tissue and longer T1s reflecting demyelination, axonal loss, and global microstructural medullary disorganization of myelopathy. The present study illustrates the potentialities of the MP2RAGE sequence in the context of patients with DCM, permitting medullary T1 mapping with valuable pathologic changes. The main findings related to the stenosis grading; clinical severity; and SC, GM, and WM ROIs are summarized in the Table and discussed further below.

\section{Clinical Correlation of Spinal Cord T1 Mapping}

A similar pattern of SC T1 mapping was found in the 3 clinical groups, shifted according to the severity of their symptoms (Fig 4). If occurrence of critical changes in T1 values at the level of maximal compression was not surprising, more remarkable were the important modifications of the T1 mapping at distal levels, simultaneously above and below the main compression. While standard anatomic MR imaging sequences primarily showed focal alterations at compressed medullary levels, the quantitative MP2RAGE sequence emphasized a more diffuse structural deterioration of the SC, potentially related to Wallerian degeneration, proximal and distal to the compression zone. ${ }^{38}$

\section{Anatomic Considerations of Spinal Cord T1 Mapping}

In healthy subjects, the T1 mapping of the SC presented an inflection of the curve centered over the vertebral $\mathrm{C} 4$ level that was also observed in the mild DCM group, and in a less obvious shape in the moderate-to-severe DCM group (Fig 4). These lower T1 values at $\mathrm{C} 4$ could potentially be correlated to the upper limit of the cervical SC enlargement, anatomically corresponding to the motor output and sensory input of the upper limbs ${ }^{39}$ and thus to the medullary zone where the bundles of WM are the most important and compact. Further studies according to the spinal levels (rather than vertebral) could be considered. ${ }^{40}$ The distribution of T1 values according to the vertebral levels in WM was lower than that in the anterior GM in healthy subjects and patients with mild DCM (Fig 6), as previously reported. ${ }^{41}$ This observation conforms to the bundles-network nature of the $\mathrm{WM}$, with strong preferential orientation and functional organization in tracts in the SC. ${ }^{42}$ In contrast, GM is characterized by a more lamellar and vascularized structure, containing the perikaryons of the medullary neurons. ${ }^{43}$ Regarding 
homogenization of WM and anterior GM values in the moderate-to-severe DCM group, it could indicate a medullary alteration predominant on WM in the more severe clinical cases, presumably related to important demyelination phenomena. The statistical comparisons of the WM and anterior GM T1 mapping among the different clinical groups at the $\mathrm{C} 1, \mathrm{Cmax}$, and C7 levels provided close results to those estimated in the SC analysis (Table). Nevertheless, these conclusions should be accepted with caution because of both the persistent risk of partial volume effects and probabilistic estimations of the segmentation between WM and GM, especially in the atrophic and compressed SC of the patients with DCM.

\section{Radiologic Correlation of Spinal Cord T1 Mapping}

The presence of T2 hypersignal was associated with increased $\mathrm{T} 1$ values at the level of maximal compression. This observation appeared consistent because both increased T1 values and the presence of T2 hypersignal may be linked to increased water content in extracellular space, inducing an increase in both T1- and T2-relaxation times. However, T2 hyperintensity in DCM (including our patients) is usually focal and located at the level of compression, as opposed to the diffuse medullary alterations demonstrated by $\mathrm{T} 1$ mapping (reaching the $\mathrm{C} 1$ level). Furthermore, T2 hyperintensities were associated with large global T1 values (Online Supplemental Data), but this association was not systematic, nor reciprocal. Consequently, this has not been further investigated but would probably require an analysis in a larger cohort.

Statistical analysis of the SC T1 mapping according to the severity of the spinal canal stenosis (Fig 3) showed a significant difference in $\mathrm{T} 1$ values between noncompressed levels (grade 0) in healthy subjects and all types of levels (grade 0-3) in patients (Fig 3). A trend toward a progressive increase of disc level T1 values according to the severity of the stenosis was observed for patients, except for grade 2 . This latter observation was probably the repercussion of the smaller number of levels classified as moderately compressed (grade 2: 13 levels) compared with the mildly (grade 1: 27 levels) and severely (grade 3: 33 levels) compressed ones. Thus, T1 mapping in our study appears as a sensitive tool to differentiate symptomatic patients from healthy subjects but fails to correlate with the severity of the spinal stenosis, despite a progressive increase in values.

In their study, Maier et $\mathrm{al}^{28}$ highlighted an inverse correlation of the $\mathrm{T} 1$ values with the severity of the spinal canal stenosis, demonstrating lower values in case of compression compared with "unaffected" segments above and below the compression. This should be further explored because MR imaging protocols, measurement techniques, and inclusion criteria (leading to different populations and pathophysiologic stages of DCM) differed.

\section{Potential Role in DCM}

T1 mapping has shown its role in the brain for multiple sclerosis, demonstrating alterations in white and gray matter (including normal-appearing tissue) and a clinical correlation with cognitive dysfunction. ${ }^{44,45}$ From the DCM perspective, the main interest of alternative MR imaging sequence studies resides in the exploration of the correlation with the preoperative clinical severity and the prediction of the postoperative outcomes. DTI parameters showed, for example, a valuable association with the mJOA score across researches; ${ }^{46}$ nevertheless, results fluctuate among other factors, according to age and vertebral levels. ${ }^{14}$ Regarding T1 mapping, measurements at both the $\mathrm{C} 1$ and $\mathrm{Cmax}$ levels appear particularly relevant because they provide both focal and diffuse information on SC tissue alterations in DCM (even in the absence of compression, as observed here in the $\mathrm{C} 1$ and $\mathrm{C} 7$ levels). Longitudinal postoperative follow-ups should now be performed to investigate these potentialities. Given current investigations and observations, T1 mapping at the level of maximal compression appears to be the most promising metric because it clearly demonstrates higher values and the most obvious group stratification/discriminating potentialities (Fig 5).

The main limitation of this study is, first, the lack of statistically significant results for the mild DCM group due to its smaller population, which thus remains the most challenging to individualize, especially from healthy controls. Another difficulty is the variable performance of the semi-automated detection and projection of the cervical SC in the compressed levels of the DCM (here performed with PropSeg ${ }^{33}$ but also tried with DeepDeg ${ }^{47}$ ) involving mandatory visual checking and manual correction in the postprocessing phase, which are sometimes time-consuming. Nonetheless, due to the feasibility and reproducibility of the protocol on the one hand ${ }^{27}$ and partial correlation of the T1 values with the clinical severity on the other, this study confirms the already encouraging results of previous experiences. ${ }^{18,28}$

Consequently, orthcoming attention to T1 mapping in DCM will have to focus on diverse key points, starting with selecting the optimal technique of measurement to standardize it. Then, larger series will have to test the robustness of the correlation with preoperative clinical severity, in particular in patients with mild DCM, identifying corresponding intervals and thresholds of T1 values. Multivariate analysis at the different cord levels should also be investigated to analyze the patients on the individual scale and to, therefore, fully demonstrate clinical use potentiality. Future prospective studies will also have to assess T1 mapping as a feasible prognostic tool for postoperative outcome, the prediction of which persists in being partially unsolved. Eventually, confrontation with other alternative MR imaging sequences will have to identify the best option in terms of sensitivity and specificity for daily practice.

\section{CONCLUSIONS}

In this preliminary study, cervical MP2RAGE-based T1 mapping in patients with DCM demonstrated diffuse medullary alterations, especially relevant at the level of maximal compression and upper (C1) levels, with promising-but-still-incomplete correlation with clinical severity. Contrary to the patients with moderate-to-severe DCM, analyses of T1 mapping of those with mild DCM notably failed to demonstrate significant differences from healthy controls, despite slightly higher T1 values. Nevertheless, SC T1 mapping appears to be a biomarker of interest, particularly concerning the clinical severity in DCM, which will now have to be explored in a number of larger series, especially including more patients with mild DCM, along with the ability to predict postoperative outcome.

AJNR Am J Neuroradiol 42:1348-57 Jul 2021 www.ajnr.org 


\section{ACKNOWLEDGMENTS}

The authors would like to thank T. Kober from Siemens for MP2RAGE sequence support, A. Massire and O. Girard for T1-postprocessing code support, as well as V. Gimenez, C. Costes, S. Confort-Gouny, V. Véla, M. Juge-Boulogne, and C. Laura for study logistics and R. Medeiros for editing the final manuscript version. The authors are also grateful to the 20 patients and 10 healthy subjects for their kind participation in this study. This work was performed within a laboratory member of France Life Imaging network (grant ANR-11-INBS0006) and was supported by Institut Carnot Star and Centre National de la Recherche Scientifique.

\section{REFERENCES}

1. Nouri A, Tetreault L, Singh A, et al. Degenerative cervical myelopathy: epidemiology, genetics, and pathogenesis. Spine (Phila Pa 1976) 2015;40:E675-93 CrossRef Medline

2. King JT, McGinnis KA, Roberts MS. Quality of life assessment with the medical outcomes study short form-36 among patients with cervical spondylotic myelopathy. Neurosurgery 2003;52:113-20; discussion 121 Medline

3. Kalsi-Ryan S, Karadimas SK, Fehlings MG. Cervical spondylotic myelopathy: the clinical phenomenon and the current pathobiology of an increasingly prevalent and devastating disorder. Neuroscientist 2013;19:409-21 CrossRef Medline

4. Nurick S. The pathogenesis of the spinal cord disorder associated with cervical spondylosis. Brain 1972;95:87-100 CrossRef Medline

5. Gore DR, Sepic SB, Gardner GM. Roentgenographic findings of the cervical spine in asymptomatic people. Spine (Phila $\mathrm{Pa}$ 1976) 1986;11:521-24 CrossRef Medline

6. Ernst CW, Stadnik TW, Peeters E, et al. Prevalence of annular tears and disc herniations on MR images of the cervical spine in symptom free volunteers. Eur J Radiology 2005;55:409-14 CrossRef Medline

7. Schneider RC, Cherry G, Pantek H. The syndrome of acute central cervical spinal cord injury. J Neurosurg 1954;11:546-77 CrossRef Medline

8. Aarabi B, Hadley MN, Dhall SS, et al. Management of acute traumatic central cord syndrome (ATCCS). Neurosurgery 2013;72 (Suppl 2):195-204 CrossRef Medline

9. Brooks NP. Central cord syndrome. Neurosurg Clin N Am 2017;28:41-47 CrossRef Medline

10. Martin AR, Tadokoro N, Tetreault L, et al. Imaging evaluation of degenerative cervical myelopathy. Neurosurg Clin N Am 2018;29:3345 CrossRef Medline

11. Tracy JA, Bartleson JD. Cervical spondylotic myelopathy. Neurologist 2010;16:176-87 CrossRef Medline

12. Nouri A, Martin AR, Mikulis D, et al. Magnetic resonance imaging assessment of degenerative cervical myelopathy: a review of structural changes and measurement techniques. Neurosurg Focus 2016;40: E5 CrossRef Medline

13. Stroman PW, Wheeler-Kingshott C, Bacon M, et al. The current state-of-the-art of spinal cord imaging: methods. Neuroimage 2014;84:1070-81 CrossRef Medline

14. Rindler RS, Chokshi FH, Malcolm JG, et al. Spinal diffusion tensor imaging in evaluation of preoperative and postoperative severity of cervical spondylotic myelopathy: systematic review of literature. World Neurosurg 2017;99:150-58 CrossRef Medline

15. Henderson E, McKinnon G, Lee TY, et al. A fast 3D looklocker method for volumetric T1 mapping. Magn Reson Imaging 1999;17:1163-71 CrossRef Medline

16. Deoni SCL, Rutt BK, Peters TM. Rapid combined T1 and T2 mapping using gradient recalled acquisition in the steady state. Magn Reson Med 2003;49:515-26 CrossRef Medline
17. Wang X, Joseph AA, Kalentev O, et al. High-resolution myocardial T1 mapping using single-shot inversion recovery fast low-angle shot MRI with radial undersampling and iterative reconstruction. Br J Radiol 2016;89:20160255 CrossRef Medline

18. Battiston M, Schneider T, Prados F, et al. Fast and reproducible in vivo T1 mapping of the human cervical spinal cord. Magn Reson Med 2018;79:2142-48 CrossRef Medline

19. Koenig SH, Brown RD, Spiller M, et al. Relaxometry of brain: why white matter appears bright in MRI. Magn Reson Med 1990;14:48295 CrossRef Medline

20. Does MD, Gore JC. Compartmental study of T1 and T2 in rat brain and trigeminal nerve in vivo. Magn Reson Med 2002;47:274-83 CrossRef Medline

21. Lancaster JL, Andrews T, Hardies LJ, et al. Three-pool model of white matter. J Magn Reson Imaging 2003;17:1-10 CrossRef Medline

22. Vymazal J, Righini A, Brooks RA, et al. T1 and T2 in the brain of healthy subjects, patients with Parkinson disease, and patients with multiple system atrophy: relation to iron content. Radiology 1999;211:489-95 CrossRef Medline

23. Gelman N, Ewing JR, Gorell JM, et al. Interregional variation of longitudinal relaxation rates in human brain at $3.0 \mathrm{~T}$ : relation to estimated iron and water contents. Magn Reson Med 2001;45:71-79 CrossRef Medline

24. Harkins KD, Xu J, Dula AN, et al. The microstructural correlates of T1 in white matter. Magn Reson Med 2016;75:1341-45 CrossRef Medline

25. Steenwijk MD,Vrenken H, Jonkman LE, et al. High-resolution T1relaxation time mapping displays subtle, clinically relevant, gray matter damage in long-standing multiple sclerosis. Mult Scler 2016;22:1279-88 CrossRef Medline

26. Lommers E, Simon J, Reuter G, et al. Multiparameter MRI quantification of microstructural tissue alterations in multiple sclerosis. Neuroimage Clin 2019;23:101879 CrossRef Medline

27. Rasoanandrianina H, Massire A, Guye M, et al. Fast and robust submillimetric 3D MP2RAGE-based T1 mapping of the entire cervical cord at 3T. NMR Biomed 2019;32(11):e4142 CrossRef Medline

28. Maier IL, Hofer S, Joseph AA, et al. Quantification of spinal cord compression using $\mathrm{T} 1$ mapping in patients with cervical spinal canal stenosis: preliminary experience. Neuroimage Clin 2019;21:101639 CrossRef Medline

29. Chiles BW, Leonard MA, Choudhri HF, et al. Cervical spondylotic myelopathy: patterns of neurological deficit and recovery after anterior cervical decompression. Neurosurgery 1999;44:762-69; discussion 769-70 CrossRef Medline

30. Tetreault L, Kopjar B, Nouri A, et al. The modified Japanese Orthopaedic Association scale: establishing criteria for mild, moderate and severe impairment in patients with degenerative cervical myelopathy. Eur Spine J 2017;26:78-84 CrossRef Medline

31. Marques JP, Kober T, Krueger G, et al. MP2RAGE, a self bias-field corrected sequence for improved segmentation and T1-mapping at high field. Neuroimage 2010;49:1271-81 CrossRef Medline

32. Maggioni M, Katkovnik V, Egiazarian K, et al. Nonlocal transformdomain filter for volumetric data denoising and reconstruction. IEEE Trans Image Process 2013;22:119-33 CrossRef Medline

33. De Leener B, Kadoury S, Cohen-Adad J. Robust, accurate and fast automatic segmentation of the spinal cord. Neuroimage 2014;98:52836 CrossRef Medline

34. De Leener B, Lévy S, Dupont SM, et al. SCT: Spinal Cord Toolbox, an open-source software for processing spinal cord MRI data. Neuroimage 2017;145:24-43 CrossRef Medline

35. De Leener B, Fonov VS, Collins DL, et al. PAM50: Unbiased multimodal template of the brainstem and spinal cord aligned with the ICBM152 space. NeuroImage 2018;165:170-79 CrossRef Medline

36. Fonov VS, Le Troter A, Taso M, et al. Framework for integrated MRI average of the spinal cord white and gray matter: the MNIPoly-AMU template. Neuroimage 2014;102:817-27 CrossRef Medline 
37. Muhle C, Metzner J, Weinert D, et al. Classification system based on kinematic MR imaging in cervical spondylitic myelopathy. AJNR Am J Neuroradiol 1998;19:1763-71 Medline

38. Dolan RT, Butler JS, O'Byrne JM, et al. Mechanical and cellular processes driving cervical myelopathy. World J Orthop 2016;7:20-29 CrossRef Medline

39. Bican O, Minagar A, Pruitt AA. The spinal cord: a review of the functional neuroanatomy. Neurol Clin 2013;31:1-18 CrossRef Medline

40. Cadotte DW, Cadotte A, Cohen-Adad J, et al. Characterizing the location of spinal and vertebral levels in the human cervical spinal cord. AJNR Am J Neuroradiol 2015;36:803-10 CrossRef Medline

41. Smith SA, Edden RAE, Farrell JAD, et al. Measurement of T1 and T2 in the cervical spinal cord at 3 Tesla. Magn Reson Med 2008;60:213-19 CrossRef Medline

42. Cho TA. Spinal cord functional anatomy. Continuum (Minneap Minn) 2015;21:13-35 CrossRef Medline
43. Santillan A, Nacarino V, Greenberg E, et al. Vascular anatomy of the spinal cord. J Neurointerv Surg 2012;4:67-74 CrossRef Medline

44. Vrenken H, Geurts JJ, Knol DL, et al. Whole-brain T1 mapping in multiple sclerosis: global changes of normal-appearing gray and white matter. Radiology 2006;240:811-20 CrossRef Medline

45. Spini M, Choi S, Harrison D. A comparison of FLAIR and T1 maps from MP2RAGE at 7T for quantifying lesion volume and its correlation to disability in multiple sclerosis. Neurology 2019;92:P5.2-026

46. Guan X, Fan G, Wu X, et al. Diffusion tensor imaging studies of cervical spondylotic myelopathy: a systemic review and meta-analysis. PLoS One 2015;10:e0117707 CrossRef Medline

47. Gros C, De Leener B, Badji A, et al. Automatic segmentation of the spinal cord and intramedullary multiple sclerosis lesions with convolutional neural networks. Neuroimage 2019;184:901-15 CrossRef Medline 